\title{
MODELAGEM DA CINÉTICA DE BIODEGRADAÇÃO DE PACLOBUTRAZOL EM DOIS SOLOS DO SEMIÁRIDO DO NORDESTE BRASILEIRO
}

Fernanda Leitão Vaz, André Maciel Netto, Antonio Celso Dantas Antonino e Antonio Cláudio Marques Afonso

Departamento de Energia Nuclear, Universidade Federal de Pernambuco, Av. Prof. Luiz Freire, 1000, Cidade Universitária, 50740-540 Recife - PE, Brasil

\section{Jean Manuel Fonseca Martins}

Laboratoire d'étude de Transferts en Hydrologie et Environnement, Univ. Grenoble I, CNRS, BP 53, 38041, Grenoble Cedex 09, França

\section{Ester Ribeiro Gouveia*}

Departamento de Antibióticos, Universidade Federal de Pernambuco, Av. Prof. Arthur de Sá, s/n, Cidade Universitária, 50740-520 Recife - PE, Brasil

Recebido em 2/2/11; aceito em 8/6/11; publicado na web em 22/7/11

\begin{abstract}
MODELING OF THE KINETICS BIODEGRADATION OF PACLOBUTRAZOL IN TWO SOILS OF THE SEMIARID NORTHEAST BRAZIL. Mathematical models can help to prevent high levels of toxic substances in soil or fruits of plants treated with pesticides and indicate that such substances should be systematically monitored. The aim of this research was to study the kinetics of paclobutrazol biodegradation by soil native bacteria using mathematical models. Three models were used to assess the kinetics of paclobutrazol biodegradation obtained experimentally. Excellent fits were obtained using dual kinetic and logistic models. The use of glycerol as additional carbon source increased the biodegradation of PBZ and consequently decreased the time required for a given PBZ initial concentration be halved.
\end{abstract}

Keywords: paclobutrazol; biodegradation; modeling.

\section{INTRODUÇÃO}

O Brasil é o terceiro maior exportador mundial de manga, após o México e a Índia, com cerca de 840 mil toneladas. Essa posição no ranking mundial se deve, principalmente, às ótimas condições ambientais da região associadas a modernas tecnologias de produção, como o uso de fitorreguladores. ${ }^{1} \mathrm{O}$ regulador de crescimento vegetal paclobutrazol ([2RS,3RS]-1-(4-cloro-fenil)-4,4-dimetil-2-(1H-1,2,4tiazolil-1)-pentan-3-ol), ou PBZ (Figura 1), é o principal fitorregulador usado em cultura de manga. ${ }^{2}$ Ele age através da inibição de hormônios vegetais de crescimentos, as giberelinas. ${ }^{3}$

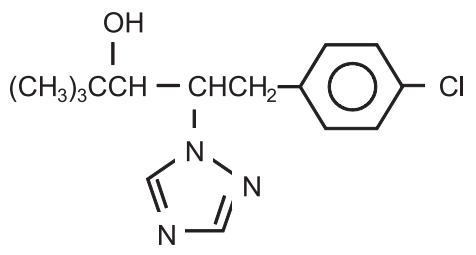

Figura 1. Fórmula estrutural da molécula de paclobutrazol

Segundo a EMBRAPA Semiárido, uma das decisões mais difíceis quando do uso do PBZ é a determinação da dosagem a aplicar. Em trabalhos experimentais são mencionadas doses sem, no entanto, especificar tamanho e condições de vigor das plantas, tipos de solo e irrigação, sendo recomendada a dose de $1 \mathrm{~g}$ por metro linear de diâmetro de copa. Entretanto, o que se verifica é que esta recomendação embora se ajuste para plantas entre 3 e 5 $\mathrm{m}$ de diâmetro, fica excessiva para plantas de diâmetro inferior e insuficiente para plantas maiores. ${ }^{4}$ Estudos têm demonstrado que em algumas épocas do ano, a utilização do paclobutrazol não

*e-mail: estergouveia@gmail.com tem apresentado respostas favoráveis à floração da mangueira, acarretando prejuízos para os produtores. ${ }^{5}$

A análise de risco de contaminação de águas subterrâneas apresentou o paclobutrazol como um agrotóxico que possui baixa mobilidade no ambiente, sendo considerado um contaminante em potencial. ${ }^{6}$ Alguns autores relataram que o paclobutrazol permanece no solo por vários anos, e que isso pode afetar o crescimento e desenvolvimento de colheitas subsequentes, principalmente pela redução do vigor vegetativo.?

A degradação microbiana, de pesticidas aplicados no solo, é o principal mecanismo que impede o acúmulo destes compostos no meio ambiente. ${ }^{8,9} \mathrm{O}$ destino de um xenobiótico do seu ponto de aplicação, pela superfície do solo e dentro do subsolo, é governado por processos interativos de adsorção, transformação, transporte e degradação. O entendimento da ciência que está por trás destes processos é uma das chaves para assegurar o seu uso ambientalmente correto. ${ }^{5}$ Também é fundamental o desenvolvimento e a validação de modelos computacionais como ferramentas preditivas nas avaliações do impacto ambiental destes produtos..$^{10,11}$

Os modelos matemáticos podem contribuir para prevenir altos níveis de substâncias tóxicas nos solos, ou nos frutos de plantas tratadas com agroquímicos e indicam que tais substâncias devem ser sistematicamente monitoradas. Os modelos matemáticos são descritos para diversas finalidade na agricultura, dentre elas, para simular a absorção de substâncias orgânicas pelas plantas, ${ }^{12,13}$ bem como o seu comportamento no solo. ${ }^{14,15}$ A determinação de equações que descrevam o processo de cinética de degradação fornece dados necessários se para estudar os efeitos negativos de um produto químico em um ecossistema.

O modelo cinético de primeira ordem tem sido largamente utilizado para descrever a cinética de dissipação de vários herbicidas naturalmente no solo, tanto em condições de campo, como no laboratório. ${ }^{16-19}$ No entanto, o uso dessa equação para a mo- 
delagem da taxa de transformação de poluentes implica que um único sistema microbiano ou enzimático esteja envolvido (com somente uma via de degradação) e que a taxa de degradação não é afetada pela toxicidade do poluente ou a adaptação microbiana (a taxa de degradação máxima não varia durante o processo). ${ }^{16}$

Para muitos compostos complexos, a cinética de primeira ordem não é adequada, pois a biodegradação desses compostos pode ocorrer com velocidades diferentes ao longo do processo. Nesse caso, resultados utilizando modelos de cinética de dupla primeira ordem têm tido bons ajustes, principalmente quando a biodegradação ocorre primeiramente numa reação mais rápida e depois segue uma degradação com uma taxa mais lenta. ${ }^{15,19}$

Outros estudos têm revelado a ampla aplicação da equação logística quadrática (ELQ) em diversas áreas ambientais, tais como, na ecologia (principalmente na descrição de processos de crescimento populacional), em relações presa-predador, em interações competitivas, no gerenciamento de fontes renováveis, na evolução da resistência a pesticidas, no controle ecológico de pestes. ${ }^{20} \mathrm{Re}$ centemente, dados sobre degradação de hipoclorito de sódio foram melhor ajustados utilizando também a equação logística. ${ }^{19}$

O objetivo deste trabalho foi estudar a cinética de biodegradação do paclobutrazol por meio de modelos já descritos na literatura e, assim, determinar o modelo que melhor representou os dados experimentais.

\section{PARTE EXPERIMENTAL}

\section{Modelagem matemática}

A degradação de compostos químicos nos solos é o resultado de uma combinação de produtos químicos e, principalmente, de eventos biológicos. ${ }^{21}$ Um modelo de degradação de primeira ordem é muitas vezes usado para simular a diminuição da massa residual de um composto químico em um sistema solo após a sua aplicação. ${ }^{22} \mathrm{Se}$ a taxa de primeira ordem for constante ou o tempo de meia-vida permanecer inalterado no processo de degradação, a massa residual da degradação química diminuirá exponencialmente com o tempo. ${ }^{16}$

Para testes de dissipação de herbicidas, o modelo de primeira ordem (Equação 1) é bastante utilizado ${ }^{16,18,23}$

$$
-\frac{d C}{d t}=k_{1} C
$$

na qual, $k_{l}$ é a constante de velocidade de primeira ordem da degradação e $C$ é a concentração do composto no tempo $t$ e é expressa em g/L. O modelo de primeira ordem tem como solução analítica a expressão obtida na Equação 2:

$$
C(t)=C_{0} e^{-k_{1} t}
$$

na qual, $C_{0}$ é a concentração inicial do composto $(\mathrm{g} / \mathrm{L})$.

Modelos de cinética de dupla primeira ordem são descritos na literatura para a degradação de alguns compostos mais complexos, visto que nesse modelo ocorrem duas reações de cinética de primeira ordem. ${ }^{15,19}$ Uma primeira reação (Equação 3), representando o início da degradação do composto mais rápido e uma segunda reação (Equação 4) quando a biodegradação se torna mais lenta.

$$
\begin{gathered}
-\frac{d C_{R}}{d t}=k_{R} C \\
-\frac{d C_{L}}{d t}=k_{L} C
\end{gathered}
$$

Integrando as equações acima com $C_{R 0}=f C_{0}$ e $C_{L 0}=(1-f) C_{0}$, a concentração de um composto no tempo pode ser descrita pela Equação 5:

$$
C(t)=C_{0}\left\{f \cdot e^{-k_{R} t}+(1-f) e^{-k_{L} t}\right\}
$$

na qual, $C(t)$ é a concentração do composto no tempo $t$ em (g/L); $C_{0}$ é a concentração inicial do composto $(\mathrm{g} / \mathrm{L}) ; C_{R 0}$ é a concentração inicial do composto que participa da reação rápida $(\mathrm{g} / \mathrm{L}) ; C_{L 0}$ é a concentração inicial do composto que participa da reação lenta $(\mathrm{g} / \mathrm{L}) ; f$ é a fração do composto que foi biodegradado atribuída à reação rápida (adimensional); $k_{R}$ é a constante de velocidade de primeira ordem da reação rápida $(1 / \mathrm{t}) ;(1-f)$ representa a fração do composto biodegradado atribuída à reação lenta (adimensional); e $k_{L}$ é a constante de velocidade de primeira ordem da reação lenta $(1 / t)$

Outra equação utilizada para descrever a biodegradação microbiana é a equação logística quadrática, ${ }^{24}$ que é dada por:

$$
\frac{d C}{d t}=k C\left(1-\frac{C}{K}\right)
$$

A solução analítica da equação logística (equação 6) é dada por:

$$
C(t)=\frac{C_{0} K e^{k t}}{K+C_{0}\left(e^{k t}-1\right)}
$$

na qual, novamente, $C(t)$ é a concentração do composto no tempo $t$ em $(\mathrm{g} / \mathrm{L}) ; C_{0}$ é a concentração inicial do composto (g/L); $K$ é a concentração final assintótica, ou seja, é a concentração em que a biodegradação do composto se estabiliza e este não é mais degradado, enquanto $k$ é uma pseudoconstante de primeira ordem e por isso está relacionada com a velocidade de degradação (1/t).

\section{Biodegradação de paclobutrazol}

Os modelos foram aplicados, utilizando o programa Sigma Plot 11.0, a resultados obtidos em estudos anteriores sobre a biodegradação de PBZ por uma cultura mista de bactérias do gênero Pseudomonas. ${ }^{25}$

Os ensaios foram realizando em batelada utilizando dois solos diferentes (Argissolo-Amarelo e Vertissolo). Os experimentos foram conduzidos em condições estéreis e não estéreis, utilizando o PBZ na sua forma pura (Sigma) e o seu produto comercial (Cultar), nas concentrações de 10 e $25 \mathrm{mg} / \mathrm{L}$. Experimentos adicionais também foram realizados utilizando apenas PBZ como fonte de carbono e PBZ e glicerol como fonte adicional de carbono.

As concentração de PBZ foram quantificadas por cromatografia líquida de alta eficiência (CLAE) segundo metodologia descrita anteriormente. ${ }^{26}$

Como não foi observada diferença significativa nos resultados dos estudos realizados neste trabalho, ${ }^{25}$ nem entre os ensaios em condições estéreis e não estéreis, nem entre PBZ (Sigma) e PBZ (Cultar), os dados desses experimentos foram agrupados como replicatas para a modelagem, somando um total de 12 réplicas. Também foram descontados os tempos lag (tempo de adaptação microbiana) referentes a 2 dias, nos experimentos com apenas PBZ como fonte de carbono e 4 dias, nos experimentos com PBZ e glicerol.

\section{RESULTADOS E DISCUSSÃO}

Os decaimentos das curvas experimentais obtidas foram ajustados a três modelos cinéticos (primeira ordem, cinética de dupla primeira ordem e logístico). 
Tabela 1. Constantes e parâmetros cinéticos da biodegradação de paclobutrazo

\begin{tabular}{|c|c|c|c|c|c|c|c|c|c|c|}
\hline & & \multicolumn{2}{|c|}{ Primeira ordem } & \multicolumn{4}{|c|}{ Dupla primeira ordem } & \multicolumn{3}{|c|}{ Logística } \\
\hline & & $\mathrm{k}$ & $\mathrm{R}$ & $\mathrm{k}_{\mathrm{R}}$ & $\mathrm{k}_{\mathrm{L}}$ & $\mathrm{R}$ & $\mathrm{f}$ & $\mathrm{K}$ & $\mathrm{k}$ & $\mathrm{R}$ \\
\hline \multirow[t]{4}{*}{ PBZ } & Argissolo 10 & 0,0315 & 0,831 & 0,1933 & 0,0037 & 0,938 & 0,3972 & 5,5157 & 0,0997 & 0,938 \\
\hline & Vertissolo 10 & 0,0344 & 0,847 & 0,1866 & 0,0027 & 0,947 & 0,4195 & 5,3889 & 0,1015 & 0,947 \\
\hline & Argissolo 25 & 0,0204 & 0,825 & 0,1822 & 0,0012 & 0,986 & 0,3624 & 15,3344 & 0,1178 & 0,986 \\
\hline & Vertissolo 25 & 0,0303 & 0,875 & 0,1795 & 0,0012 & 0,989 & 0,4009 & 14,3405 & 0,1098 & 0,988 \\
\hline \multirow[t]{4}{*}{ PBZ + Glicerol } & Argissolo 10 & 0,0452 & 0,976 & 0,2109 & 0,0277 & 0,995 & 0,3375 & 0,7824 & 0,0073 & 0,996 \\
\hline & Vertissolo 10 & 0,0526 & 0,964 & 0,1964 & 0,0223 & 0,994 & 0,4200 & 1,5618 & 0,0181 & 0,994 \\
\hline & Argissolo 25 & 0,0602 & 0,961 & 0,1453 & 0,0032 & 0,998 & 0,6817 & 4,8104 & 0,0294 & 0,998 \\
\hline & Vertissolo 25 & 0,0582 & 0,956 & 0,1614 & 0,0084 & 0,997 & 0,6226 & 5,1894 & 0,0324 & 0,997 \\
\hline
\end{tabular}

O coeficiente de correlação $(\mathrm{R})$ mostrou que o modelo de primeira ordem não se ajustou bem aos dados experimentais (Tabela 1). Isso se deu, principalmente, porque a biodegradação de paclobutrazol ocorreu em duas etapas distintas, uma mais rápida e outra mais lenta, tendendo para a estabilização da biodegradação. Isso ocorre, provavelmente, devido à complexidade da molécula de paclobutrazol, que é formada por um anel triazol e um anel cloro-benzeno e possui ainda uma cadeia carbônica aberta.

Poucos são os trabalhos na literatura que descrevam a biodegradação do paclobutrazol, e há menos trabalhos ainda sobre a modelagem desse processo. Em trabalhos prévios, alguns autores fizeram um estudo de biodegradação desse composto diretamente no solo, sem adição de micro-organismos, ou seja, apenas como a participação dos micro-organismos presentes naturalmente no solo e utilizaram um modelo de cinética de primeira ordem para ajustar os dados experimentais. Esses autores também não encontraram um bom ajuste, com um coeficiente de correlação igual a $0,93 .^{27}$

As equações de cinética de dupla primeira ordem e logística apresentaram ótimos ajustes aos dados experimentais, para os dois solos estudados (Argissolo-Amarelo e Vertissolo), bem como para as duas concentrações de PBZ utilizadas (10 e $25 \mathrm{mg} / \mathrm{L})$, tanto nos experimentos apenas com PBZ, quanto nos experimentos com PBZ e glicerol (Figuras 2-5), conforme se pode averiguar pelo coeficiente de correlação (R) (Tabela 1).

Em outros estudos foram utilizados vários modelos para descrever a degradação de hipoclorito de sódio e de uma amina alifática (alquilamina). ${ }^{19}$ Dentre os modelos analisados por estes autores, os de maior

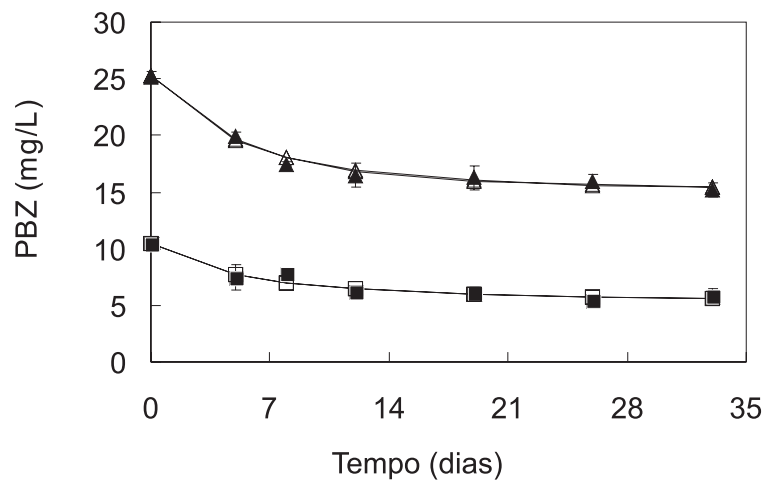

Figura 2. Modelagens cinética de dupla primeira ordem e logística aplicadas aos dados experimentais de biodegradação de paclobutrazol utilizando o mesmo como única fonte de carbono em Argissolo-Amarelo nas concentrações de PBZ de 10 e $25 \mathrm{mg} / \mathrm{L}$. - (10 mg/L) experimental; - - - - - $10 \mathrm{mg} / \mathrm{L})$ cinética dupla; - - $(10 \mathrm{mg} / \mathrm{L})$ logística; $\mathbf{\Delta}(25 \mathrm{mg} / \mathrm{L})$ experimental; - - $\triangle$ - - $(25 \mathrm{mg} / \mathrm{L})$ cinética dupla; $-\triangle-(25 \mathrm{mg} / \mathrm{L})$ logística êxito foram os de cinética de dupla primeira ordem e logístico. Por outro lado, o modelo que menos se ajustou foi o de primeira ordem. Para estes autores, o modelo escolhido para melhor se ajustar a uma degradação depende de como se sucede o processo degradativo do composto orgânico estudado.

Esse perfil observado na biodegradação do PBZ, em que a mesma é mais rápida no começo e praticamente cessa ao longo do tempo,

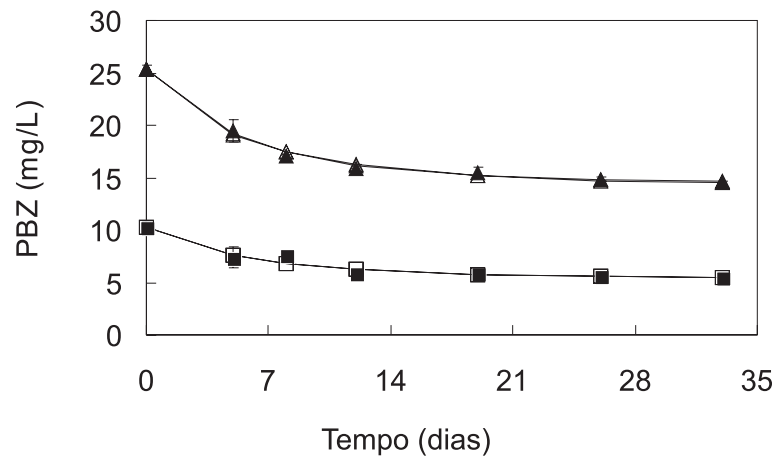

Figura 3. Modelagens cinética de dupla primeira ordem e logística aplicadas aos dados experimentais de biodegradação de paclobutrazol utilizando o mesmo como única fonte de carbono em Vertissolo nas concentrações de PBZ de 10 e $25 \mathrm{mg} / \mathrm{L}$. - (10 mg/L) experimental; - - - - (10 mg/L) cinética dupla; - $\square-(10 \mathrm{mg} / \mathrm{L})$ logística; $\boldsymbol{\Delta}(25 \mathrm{mg} / \mathrm{L})$ experimental; - - $\triangle--(25 \mathrm{mg} / \mathrm{L})$ cinética dupla; $-\triangle-(25 \mathrm{mg} / \mathrm{L})$ logística

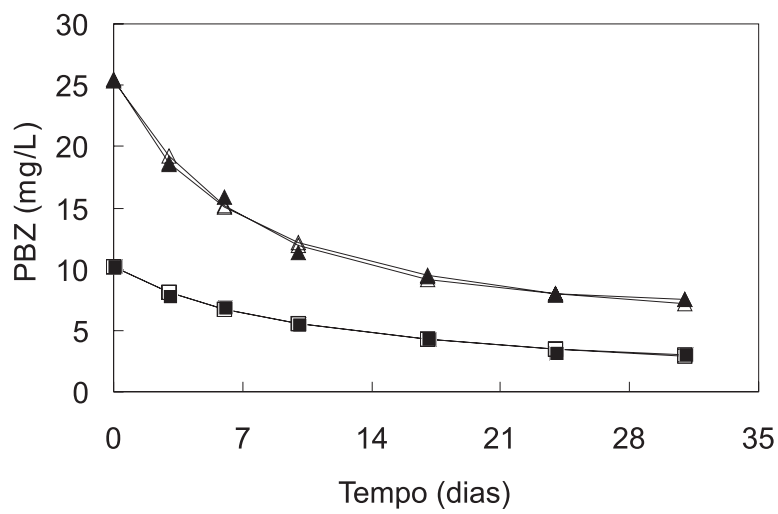

Figura 4. Modelagens cinética de dupla primeira ordem e logística aplicadas aos dados experimentais de biodegradação de paclobutrazol utilizando PBZ adicionado de glicerol como fontes de carbono em Argissolo-Amarelo nas concentrações de $10 \mathrm{mg} / \mathrm{L}$ de PBZ : $50 \mathrm{mg} / \mathrm{L}$ de glicerol e $25 \mathrm{mg} / \mathrm{L}$ de PBZ: $125 \mathrm{mg} / \mathrm{L}$ de glicerol. - (10 mg/L) experimental; - - $\square$ - - (10 mg/L) cinética dupla; - $-(10 \mathrm{mg} / \mathrm{L})$ logística; $\mathbf{\Delta}(25 \mathrm{mg} / \mathrm{L})$ experimental; - - $\triangle--(25 \mathrm{mg} / \mathrm{L})$ cinética dupla; $-\triangle-(25 \mathrm{mg} / \mathrm{L})$ logística 


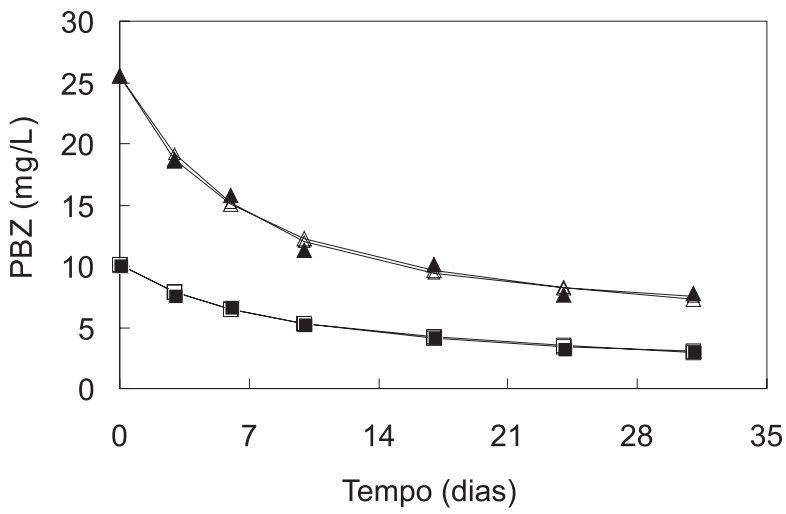

Figura 5. Modelagens cinética de dupla primeira ordem e logística aplicadas aos dados experimentais de biodegradação de paclobutrazol utilizando PBZ adicionado de glicerol como fontes de carbono em Vertissolo nas concentrações de $10 \mathrm{mg} / \mathrm{L}$ de PBZ : $50 \mathrm{mg} / \mathrm{L}$ de glicerol e $25 \mathrm{mg} / \mathrm{L}$ de PBZ: $125 \mathrm{mg} / \mathrm{L}$ de glicerol. $\square(10 \mathrm{mg} / \mathrm{L})$ experimental; - - - - $(10 \mathrm{mg} / \mathrm{L})$ cinética dupla; - $-(10$ $\mathrm{mg} / \mathrm{L})$ logística; $\mathbf{\Delta}(25 \mathrm{mg} / \mathrm{L})$ experimental; - - $\triangle$ - - $(25 \mathrm{mg} / \mathrm{L})$ cinética dupla; $-\triangle-(25 \mathrm{mg} / \mathrm{L})$ logística

foi o que fez com que não se obtivesse um bom ajuste com uma cinética de primeira ordem simples, isto é, com apenas uma velocidade. Entretanto, esse mesmo perfil foi o responsável pelo ótimo ajuste obtido quando se utilizaram as equações de cinética de dupla primeira ordem e logística. Esse decaimento característico também foi observado em ensaios de biodegradação de PBZ, realizados em outros estudos. ${ }^{27,28} \mathrm{O}$ PBZ não é mais degradado, provavelmente, por alguma incapacidade das bactérias de produzir enzimas necessárias para continuar essa degradação. Possivelmente, a biodegradação natural desse composto no solo pode levar mais tempo que o observado em laboratório, porém através de uma sucessão ecológica microbiana essa biodegradação pode chegar a $100 \%$.

O coeficiente $f$ obtido através do modelo de cinética de dupla primeira ordem, que corresponde à fração de PBZ que foi biodegradada durante a reação rápida, foi aproximadamente 0,4 nos experimentos que utilizaram apenas PBZ como fonte de carbono (Figuras 2 e 3) e nos experimentos com PBZ (10 mg/L) e glicerol (50 mg/L) (Figuras 4 e 5). Isso significa que $40 \%$ da biodegradação de PBZ foi realizada mais rapidamente, numa taxa $k_{R}$ que ficou em torno de $0,19 \mathrm{~h}^{-1}$, enquanto que $60 \%$ da biodegradação ocorreu numa taxa mais lenta, $k_{L}$. Já nos experimentos utilizando as concentrações de $25 \mathrm{mg} / \mathrm{L}$ de PBZ e $125 \mathrm{mg} / \mathrm{L}$ glicerol, o coeficiente $f$ foi de 0,68 e 0,62 , para os solos Argissolo-Amarelo e Vertissolo, respectivamente (Figuras $4 \mathrm{e}$ 5). De acordo com este resultado, $68 \%$ da biodegradação de $P B Z$ em Argissolo-Amarelo ocorreu na fase rápida e $62 \%$ da biodegradação em Vertissolo correspondeu a esta mesma fase.

Através do modelo de cinética de dupla primeira ordem pode-se estimar quando a biodegradação total poderá ser alcançada. Consequentemente, por esse modelo também é possível calcular o tempo necessário para uma dada concentração inicial de PBZ se reduzir à metade $\left(\mathrm{T}_{50}\right)$.

Para os ensaios em que foi utilizado apenas o PBZ como fonte de carbono na concentração inicial de $10 \mathrm{mg} / \mathrm{L}, \mathrm{o} \mathrm{T}_{50}$ foi estimado em 50,7 dias para o Argissolo-Amarelo e 57,5 dias para o Vertissolo. Já para a concentração de $\mathrm{PBZ}$ de $25 \mathrm{mg} / \mathrm{L}, \mathrm{o}_{50}$ calculado aumentou para 200 e 150,6 dias, para o Argissolo-Amarelo e o Vertissolo, respectivamente. $\mathrm{Na}$ literatura considera-se o $\mathrm{T}_{50}$ do paclobutrazol no solo em torno de 0,5 dias a 1 ano..$^{29}$

Os $\mathrm{T}_{50}$ estimados para os experimentos em que o glicerol foi utilizado como fonte adicional de carbono ao PBZ apresentaram menores valores se comparados aos experimentos em que foi utilizado apenas PBZ como fonte de carbono. Para o Argissolo-Amarelo o $\mathrm{T}_{50}$ ficou em torno de 12,1 dias e para o Vertissolo 11,2 dias, quando o PBZ foi utilizado na concentração inicial de $10 \mathrm{mg} / \mathrm{L}$. Nos experimentos em que a concentração inicial de PBZ foi de $25 \mathrm{mg} / \mathrm{L}$, o $\mathrm{T}_{50}$ foi de aproximadamente 8,8 dias para o Argissolo-Amarelo e 8,9 dias para o Vertissolo. Esses resultados revelam que o glicerol, quando usado como fonte adicional de carbono, contribui para diminuir o $\mathrm{T}_{50}$ do PBZ. Para maiores concentrações de glicerol, o $\mathrm{T}_{50}$ diminui ainda mais.

Alguns trabalhos têm demonstrado esta habilidade do glicerol de aumentar a biodegradação de PBZ. Foi verificado, em outros experimentos, que a degradação de PBZ por Pseudomonas aeruginosa BB2-572 aumentou significativamente quando, além deste composto, os meios continham glicerol como fonte de carbono adicional..$^{30,31} \mathrm{Em}$ estudos com bactérias isoladas do solo, foi observada a degradação de $75 \%$ do paclobutrazol quando foi adicionado glicerol ao meio, contra apenas $47 \%$ de biodegradação sem a adição de glicerol. ${ }^{28}$

Vale ressaltar que o $\mathrm{T}_{50}$ só foi obtido experimentalmente nos ensaios com PBZ adicionado de glicerol, nas duas concentrações e para os dois solos. Nos ensaios com apenas PBZ como fonte de carbono, os $\mathrm{Ts}_{50}$ foram estimados através do modelo de cinética de dupla primeira ordem (modelo que melhor representou os dados experimentais). Os $\mathrm{Ts}_{50}$ obtidos, tanto experimentalmente, quanto através do modelo de cinética de dupla primeira ordem, para os experimentos com PBZ e glicerol foram correspondentes. De acordo com esta observação, é possível crer que os $\mathrm{Ts}_{50}$ estimados por este mesmo modelo, para as outras situações estudadas neste trabalho, estejam provavelmente próximos do que seria observado.

Ao contrário do modelo de cinética de dupla primeira ordem, o modelo logístico não permite a estimativa do tempo de biodegradação total, nem do $\mathrm{T}_{50}$, quando estes dados não foram determinados experimentalmente, pois o mesmo tende a uma curva assintótica não chegando a valores menores que o coeficiente $K$ (concentração final) encontrado. Estes dados são de grande importância do ponto de vista ambiental, uma vez que se a biodegradação de PBZ seguir o proposto pelo modelo logístico o decaimento total e, em alguns casos, nem o decaimento à metade serão alcançados, ficando assim um residual acumulado no solo.

\section{CONCLUSÕES}

Os modelos de cinética de dupla primeira ordem e logístico se ajustaram bem aos dados experimentais. Por meio do modelo cinético de dupla primeira ordem foi possível estimar o $\mathrm{T}_{50}$ para a biodegradação de PBZ. O glicerol utilizado como fonte adicional de carbono aumentou a biodegradação de PBZ e, consequentemente, diminuiu o $\mathrm{T}_{50}$ desse composto.

\section{REFERÊNCIAS}

1. http://www.cpatsa.embrapa.br:8080/public_eletronica/downloads/ SDC189.pdf, acessada em Julho 2011.

2. Srivastav, M.; Kishor, A.; Dahuja, A.; Sharma, R. R.; Sci Hortic. 2010 , 125,785 .

3. Jaleel, C. A.; Manivannan, P.; Kishorekumar, K.; Sankari, S.; Panneerselvam, R.; Process Biochem. 2007, 42, 1566.

4. http://www.cpatsa.embrapa.br/public_eletronica/downloads/OPB28.pdf, acessada em Julho 2011.

5. Silva, C. M. M. S.; Fay, E. F.; Jonsson, C. M. Em Impacto Ambiental do Regulador de Crescimento Vegetal Paclobutrazol; Silva, C. M. M. S.; Fay, E. F., eds.; Jaguariúna: EMBRAPA Meio Ambiente, 2003, p. 9-14.

6. Milfont, M. L.; Antonino, A. C. D.; Martins, J. M. F.; Netto, A. M.; Gouveia, E. R.; Correa, M. M.; Rev. Bras. Ciênc. Solo 2008, 32, 2165. 
7. Jackson, M. J.; Line, M. A.; Hasan, O.; Soil Biol. Biochem. 1996, 28, 1265.

8. Araújo, T. M. R.; Dissertação de Mestrado, Universidade Estadual do Norte Fluminense, Brasil, 2006.

9. Arbeli, C.; Fuentes, C. L.; Crop Protec. 2007, 26, 1733.

10. Vázquez-Rodríguez, G.; Youssef, C. B.; Waissman-Vilanova, J.; Chem. Eng. J. 2006, 117, 245.

11. Karahan, O.; Olmez-Hanci, T.; Arslan-Alaton, I.; Orhon, D.; Bioresour Technol. 2010, 101, 8058.

12. Fujisawa, T.; Ichise, K.; Fukushima, M.; Katagi, T.; Takimoto, Y.; $J$. Agric. Food Chem. 2002, 50, 532.

13. Trapp, S.; Rasmussen, D.; Samsøe-Petersen, L.; SAR QSAR Environ. Res. 2003, 14, 17.

14. Martins, J.; Tese de Doutorado, Université Joseph Fourier, França, 1993.

15. Gang, D. C.; Clevenger, T. E.; Banerji, S. K.; J. Environ. Informatics 2003, 1, 21.

16. Martins, J. M. F.; Mermoud, A.; J. Contam. Hydrol. 1998, 33, 187.

17. Rigitano, R. L. O.; Alves, A. D.; Souza, J. C.; Franco, A. A.; Ciência e Agrotecnologia 2001, 25, 1295.

18. Scorza Júnior, R. P.; Rigitano, R. L. O.; Boletim de pesquisa e desenvolvimento - EMBRAPA 2009, 49, 1.

19. López-Galindo, C.; Garrido, M. C.; Casanueva, J. F.; Nebot, E.; Sci. Total Environ. 2010, 408, 1779.
20. Edelstein-Keshet, L.; Mathematical Models in Biology, Random House: New York, 1988.

21. Wu, J.; Nofziger, D. L.; J. Environ. Qual. 1999, 28, 92.

22. Dykaar, B. B.; Kitanidis, P. K.; Water Resour. Res. 1996, 32, 307.

23. Chirnside, A. E. M.; Ritter, W. F.; Radosevich, M.; Soil Biol. Biochem. 2009, 41, 2484.

24. Varela, H.; Torresi, R. M.; Gonzalez, E. R.; Quim. Nova 2002, 25, 99.

25. Vaz, F. L.; Tese de Doutorado, Universidade Federal de Pernambuco, Brasil, 2011.

26. Vaz, F. L.; Milfont, M. L. B.; Souto-Maior, A. M.; Gouveia, E. R.; Quim. Nova 2007, 30, 281.

27. Silva, C. M. M. S.; Fay, E. F.; Vieira, R. F.; Pesq. Agropec. Bras. 2003, 38,1223 .

28. Vaz, F. L.; Dissertação de Mestrado, Universidade Federal de Pernambuco, Brasil, 2006.

29. Worthing, C. R.; Hance, R. H.; The pesticide manual, $11^{\text {th }}$ ed., The British Crop Protection Council: Farnham, 1994.

30. Figueiredo, C. M.; Gondim, M. V. S.; Gouveia, E. R.; Resumos do III Congresso Nacional de Meio Ambiente, Salvador, Brasil, 2004.

31. Gondim, M. V. S.; Figueiredo, C. M.; Gouveia, E. R.; Resumos do III Congresso Nacional de Meio Ambiente, Salvador, Brasil, 2004. 